\title{
Data Management on Non-Volatile Memory
}

\author{
Joy Arulraj \\ Georgia Institute of Technology \\ arulraj@gatech.edu
}

\begin{abstract}
We are at an exciting point in the evolution of memory technology. Device manufacturers have created a new nonvolatile memory (NVM) technology that can serve as both system memory and storage. NVM supports fast reads and writes similar to volatile memory, but all writes to it are persistent like a solid-state disk. The advent of NVM invalidates decades of design decisions that are deeply embedded in today's database management systems (DBMSs). These systems are unable to take full advantage of NVM because their internal architectures are predicated on the assumption that memory is volatile. With NVM, many of the components of today's DBMSs are unnecessary and will degrade the performance of data-intensive applications. Thus, the best way to resolve these shortcomings is by designing a new system explicitly tailored for NVM.
\end{abstract}

In this talk, I will present our research on the design and development of an NVM DBMS, called Peloton [1,2]. Peloton's architecture shows that the impact of NVM spans across all the layers of the DBMS. I will first introduce write-behind logging, an NVM-centric protocol that improves the availability of the database system by two orders-of-magnitude compared to the widely-used writeahead logging protocol. I will then present the BzTree, an NVM-centric index data structure that illustrates how to simplify programming on NVM. In drawing broader lessons from this work, I will argue that all types of software systems, including file systems, machine-learning systems, and key-value stores, are amenable to similar architectural changes to achieve high performance and availability on NVM.

\section{Author Keywords}

Non-Volatile Memory, Logging and Recovery, Storage Management, Buffer Management, Indexing
Permission to make digital or hard copies of part or all of this work for personal or classroom use is granted without fee provided that copies are not made or distributed for profit or commercial advantage and that copies bear this notice and the full citation on the first page. Copyrights for third-party components of this work must be honored. For all other uses, contact the Owner/Author.

SIGMOD '19, fune 30-fuly 5, 2019, Amsterdam, Netherlands.

(C) 2019 Copyright is held by the owner/author(s).

ACM ISBN 978-1-4503-5643-5/19/06.

DOI: https://doi.org/10.1145/3299869.3328523

\section{BIOGRAPHY}

Joy Arulraj is an assistant Professor of Computer Science at Georgia Institute of Technology. He received his Ph.D. from Carnegie Mellon University in 2018, advised by Andy Pavlo. His doctoral research focused on the design and implementation of non-volatile memory database management systems. This work was conducted in collaboration with the Intel Science and Technology Center for Big Data, Microsoft Research, and Samsung Research.

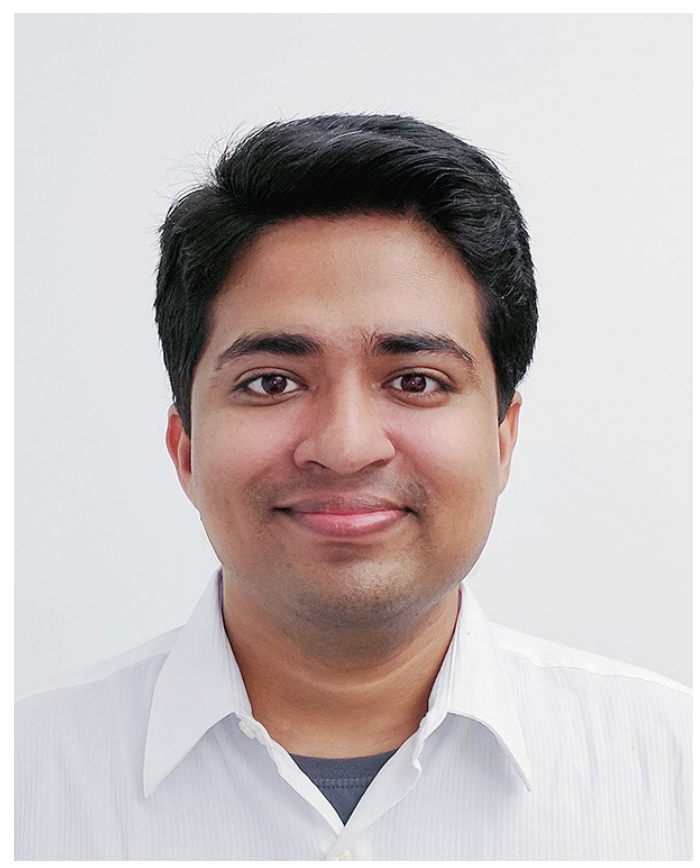

\section{REFERENCES}

[1] Peloton DBMS. http://pelotondb.org

[2] J. Arulraj and A. Pavlo, Non-Volatile Memory Database Management Systems, In Synthesis Lectures on Data Management, volume 14, pages 1-191, 2019. 